\title{
Gic1 is a novel heterochromatin boundary protein in vivo
}

\author{
Risa Mitsumori ${ }^{1}$, Kaori Shinmyozu ${ }^{2}$, Jun-ichi Nakayama ${ }^{3}$, \\ Hiroyuki Uchida ${ }^{1}$ and Masaya Oki ${ }^{1,4,5 *}$ \\ ${ }^{1}$ Department of Applied Chemistry \& Biotechnology, Graduate School of Engineering, University of Fukui, \\ Fukui 910-8507, Japan \\ ${ }^{2}$ Proteomics Support Unit Center for Developmental Biology, RIKEN, Kobe 650-0047, Japan \\ ${ }^{3}$ Graduate School of Natural Sciences, Nagoya City University, Nagoya 467-8501, Japan \\ ${ }^{4}$ Research and Education Program for Life Science, University of Fukui, Fukui 910-8507, Japan \\ ${ }^{5}$ PRESTO, Japan Science and Technology Agency (JST), Kawaguchi 332-0012, Japan
}

(Received 29 October 2015, accepted 24 December 2015; J-STAGE Advance published date: 10 June 2016)

In Saccharomyces cerevisiae, HMR/HML, telomeres and ribosomal DNA are heterochromatin-like regions in which gene transcription is prevented by the silent information regulator (Sir) complex. The Sir complex (Sir2, Sir3 and Sir4) can spread through chromatin from the silencer. Boundaries prevent Sir complex spreading, and we previously identified 55 boundary genes among all $\sim 6,000$ yeast genes. These boundary proteins can be distinguished into two types: those that activate transcription to prevent spreading of silencing, and those that prevent gene silencing by forming a boundary. We selected 44 transcription-independent boundary proteins from the 55 boundary genes by performing a one-hybrid assay and focused on GIC1 (GTPase interaction component 1). Gic1 is an effector of Cdc42, which belongs to the Rho family of small GTPases, and has not been reported to function in heterochromatin boundaries in vivo. We detected a novel boundary-forming activity of Gic1 at HMR-left and telomeric regions by conducting a chromatin immunoprecipitation assay with an anti-Sir3 antibody. We also found that Gic1 bound weakly to histones in two-hybrid analysis. Moreover, we performed domain analysis to identify domain(s) of Gic1 that are important for its boundary activity, and identified two minimum domains, which are located outside its Cdc42-binding domain.

Key words: boundary, euchromatin and heterochromatin, gene silencing, GIC1, Saccharomyces cerevisiae

\section{INTRODUCTION}

$H M R / H M L$, telomeres and ribosomal DNA (rDNA) are heterochromatic regions in Saccharomyces cerevisiae. Polymerization of the silent information regulator (Sir) proteins Sir2, Sir3 and Sir4 is required to achieve and maintain transcriptional silencing (Loo and Rine, 1995; Sun et al., 2011). Sir2 is a NAD+-dependent histone deacetylase and deacetylates the $\mathrm{N}$-terminal region of histone H3/4 (Tanny et al., 1999; Imai et al., 2000; Ito et al., 2000; Landry et al., 2000). The Sir complex (Sir2/3/4) spreads from the silencer $(H M L$-E, $H M L-\mathrm{R}, H M R$-E and $H M R-\mathrm{R})$ at $H M R / H M L$ and inhibits transcriptional activity (Triolo and Sternglanz, 1996; Moazed, 2001), while at telomeres the complex spreads from TG1-3 repeats. Silencing at rDNA does not require Sir3 or

Edited by Hiroshi Iwasaki

* Corresponding author. E-mail: ma4sa6ya@u-fukui.ac.jp DOI: http://doi.org/10.1266/ggs.15-00070
Sir4, in contrast to that at telomeres and $H M R / H M L$. However, Sir2 is required for silencing at rDNA regions (Rusche et al., 2003; Moazed et al., 2004).

However, spreading of the silencing region must be stopped to allow the transcription of essential genes. Thus, a boundary must exist to prevent spreading of gene silencing and protect gene activity. Two types of boundary models have been reported (Oki and Kamakaka, 2002). One boundary type is specific to the DNA sequence and protects against spreading of gene silencing. For example, tDNA near to the $H M R$-left site and the STAR sequence at telomeres are specific DNA sequences for boundaries. tDNA is a well-known boundary in $S$. cerevisiae (Donze et al., 1999; Donze and Kamakaka, 2001; Oki and Kamakaka, 2005). tDNA binds to TFIIIC, a component of DNA polymerase III, and can prevent spreading of silencing (Simms et al., 2008; Valenzuela et al., 2009). In telomeres, the $S T A R$ sequence, which consists of the $S T R$ sequence in the X-region and Y $-S T A R$ in the 3 terminal region of the $Y$ element, acts as a spe- 
cific DNA sequence at boundaries (Fourel et al., 1999). Tbf1 and Reb1 can bind to the STAR sequence and prevent spreading of silencing. The other model is the DNA sequence-independent model, in which proteins protect against gene silencing. Sas2 is one such well-known protein (Kimura et al., 2002; Suka et al., 2002). Sas2 is a histone acetyltransferase (HAT) and competes with Sir2, a NAD+-dependent histone deacetylase. They form a boundary and change the chromatin structure from heterochromatin to euchromatin and vice versa.

To identify other boundary proteins, our group previously developed a genome-wide screening system (Oki et al., 2004). This system detected 55 boundary proteins among the total of $\sim 6,000 \mathrm{~S}$. cerevisiae genes. These 55 genes are classified into eight groups (Histone modifications, SWI/SNF, TFIID, Mediator, Transcription factors, Cell cycle, Other, and Unknown). We previously investigated the function of some of these 55 proteins in boundary formation. For example, Sgf29 belongs to the Histone modifications group and is a component of the Spt-Ada-Gcn5-acetyltransferase (SAGA) complex (Kamata et al., 2014). It is conserved from human to yeast and is important for maintaining transcription. Domain analysis revealed that Sgf29 has two boundary elements, and a tag fusion system revealed that these two elements form a complex with Gen5, Ada1 and Ada3. We also analyzed another boundary protein, Ycr076c/Fub1, which belongs to the Unknown category and interacts with multiple subunits of the $20 \mathrm{~S}$ proteasome core particle. We found that the proteasome and Fub1 work together to establish a boundary, using a proteasome factor-deficient strain (Hatanaka et al., 2011).

In this study, we investigated a new boundary element to demonstrate DNA sequence-independent boundary formation and to identify a new form of boundary organization. We isolated proteins with DNA sequenceindependent boundary function among the 55 boundary proteins using a one-hybrid assay and investigated the boundary function of Gic1 (GTPase interaction component 1). Gic1 did not activate transcription, and we sought to understand its relationship with the silencing region. Gic1 is an effector of Cdc42, a Rho family small GTPase (Johnson, 1999), and is involved in cytokinesis and bud growth, localization, and appearance in yeast (Brown et al., 1997; Chen et al., 1997; Hofken and Schiebel, 2004). Recent studies reported that Gic1 is involved in septin recruitment, and in ring formation and dissociation (Iwase et al., 2006; Lindsey et al., 2010; Sadian et al., 2013). Gic1 has a homologous protein, Gic2 (GTPase interaction component 2), which is also an effector of Cdc42 (Jaquenoud and Peter, 2000). Gic1 and Gic2 colocalize with $\mathrm{Cdc} 42$ as cell polarity is established during the cell cycle, and during mating in response to pheromones. The double mutant gic1/gic2 $\Delta$ has a temperaturesensitive defect in polarity establishment, with perturbed actin cytoskeleton organization (Brown et al., 1997; Chen et al., 1997). Gic1 and Gic2 have a CRIB (Cdc42/Rac interactive binding) domain, which assists their transport to cell membranes and binding to Cdc42, and is conserved from human to yeast (Burbelo et al., 1995). Another study reported a new Gic1 domain, the BR (basic-rich) domain, which is located at the $\mathrm{N}$ terminus of the CRIB domain and is basic and hydrophobic (Takahashi and Pryciak, 2007). A fluorescence assay of BR-EGFP revealed that the BR domain localizes in both the nucleus and cytoplasm. Interestingly, the nuclear localization of Gic1 depends on the basic residues of the BR domain.

In this study, we report a novel function of Gic1 in boundary formation at heterochromatic regions.

\section{MATERIALS AND METHODS}

Strains The yeast strains used in this study are listed in Supplementary Table S1. Target genes were replaced by the KanMAX gene (Knockout Strain Collection, Open Biosystems). Yeast cells were transformed with PCR products amplified from genomic DNA.

Plasmids The plasmids used in this study are listed in Supplementary Table S2. All domains were obtained by PCR amplification and cloned into the pGBK-RC plasmid. In the boundary domain-deleted mutant, pFOM781-784 was generated using pFOM56 (GBD-Gic1p) by self-PCR and checked by sequencing. The primers are listed in Supplementary Table S3.

One- and two-hybrid assays To determine the transcriptional activity and cooperation of Gic1, histones and Sir proteins, a $\beta$-galactosidase activity assay was performed as described previously (Reynolds et al., 2001). Yeast cells were transformed with $G B D, G B D$-fusion genes, or $G B D$-fusion genes and $G A D$-fusion genes and then grown in synthetic dextrose (SD) medium containing adenine, leucine, lysine, uracil and histidine (but lacking tryptophan), or adenine, lysine, uracil and histidine (but lacking leucine and tryptophan) for 2 or 3 days at $30{ }^{\circ} \mathrm{C}$. Inoculated overnight cultures grew to mid-log phase $\left(\mathrm{OD}_{600}=1.5-2.0\right)$. Cells collected from $1 \mathrm{ml}$ of each medium were resuspended in $1 \mathrm{ml}$ of $\mathrm{Z}$ buffer $(60 \mathrm{mM}$ $\mathrm{Na}_{2} \mathrm{HPO}_{4} \cdot 7 \mathrm{H}_{2} \mathrm{O}, 40 \mathrm{mM} \mathrm{NaH} \mathrm{PO}_{4} \cdot \mathrm{H}_{2} \mathrm{O}, 10 \mathrm{mM} \mathrm{KCl}, 1 \mathrm{mM}$ $\mathrm{MgSO}_{4} 7 \mathrm{H}_{2} \mathrm{O}$, and $50 \mathrm{mM} 2$-mercaptoethanol) and placed on ice. Thereafter, $0.2 \mathrm{ml}$ of $4 \mathrm{mg} / \mathrm{ml}$ ONPG was added and samples were placed in a water bath at $28^{\circ} \mathrm{C}$. When a medium-yellow color developed, the reaction was stopped by adding $0.5 \mathrm{ml}$ of $1 \mathrm{M} \mathrm{Na}_{2} \mathrm{CO}_{3}$, the time was noted, and $\mathrm{OD}_{420}$ was measured. Units were calculated using the following equation: $\mathrm{U}=1000 \times\left(\mathrm{OD}_{420}\right)-(1.75 \times$ $\left.\mathrm{OD}_{600}\right) / \mathrm{T} \times \mathrm{V} \times \mathrm{OD}_{600}$. This was calculated three times independently and normalized by the value of the gene with the highest activity. 
Chromatin immunoprecipitation (ChIP) assay To determine the boundary activity of Gic1 in vivo, a ChIP assay was performed as described previously (Hatanaka et al., 2011). Cells were harvested from $200 \mathrm{ml}$ of YPD medium at log phase $\left(\mathrm{OD}_{600}=2.0\right)$, washed once with phosphate-buffered saline, and crosslinked with $1 \%$ formaldehyde for $30 \mathrm{~min}$ at room temperature. Immunoprecipitation (IP) was performed using a rabbit polyclonal anti-Sir3p antibody (a gift from Dr. R. T. Kamakaka). The amount of DNA immunoprecipitated was analyzed using the Applied Biosystems SYBR Green RT-PCR system. Each immunoprecipitation was normalized by the MAT control. The primers are listed in Supplementary Table S4.

Mating assay To identify the minimum domain(s) of Gic1 required for its boundary function, a mating assay was performed as described previously (Oki et al., 2004). Yeast cells were transformed with $G B D$ and $G B D$-fusion genes and grown in SD medium lacking tryptophan at $30^{\circ} \mathrm{C}$ for 3 days. Single colonies were streaked onto SD plates containing mating tester lawns with an $\mathrm{OD}_{600}$ of 2.0. Plates were incubated at $30{ }^{\circ} \mathrm{C}$ for 3 days.

\section{RESULTS}

Selection of Gic1 in the screening of boundary proteins By performing a mating assay, we previously identified 55 genes related to boundary formation among 6,000 genes in S. cerevisiae (Oki et al., 2004). In this method, the Gal4-binding sequence was inserted between $\mathrm{E}$ silencer and the reporter gene $a 1$ in the silencing region of $H M R$, and we determined whether each GBD-fusion protein had boundary function. However, some of the 55 boundary proteins were predicted to have transcriptional activity. First, to exclude proteins that had a transcription-associated boundary among the 55 proteins identified, we performed a one-hybrid assay to identify proteins with low transcriptional activity that had a transcriptionindependent boundary (Fig. 1). This method detected $\beta$ galactosidase activity and calculated transcriptional activity. We used a threshold level of $\beta$-galactosidase activity of around 0.2 to permit visual appraisal of the change in color, and identified 44 genes with a transcription-independent boundary function.

We selected GIC1, which belongs to categories other than those of Histone modifications, Transcription factors, Cell cycle or Mediator, for boundary screening. Gic1 is known to bind to the bud of yeast (Hofken and Schiebel, 2004). However, to our knowledge, Gic1 has not been reported to be associated with boundary formation; therefore, we expected to identify a new boundary formation system by studying Gic1.

Gic1 has boundary activity at $H M R$ and $T e l-V R$ in vivo Because we used an artificial $G B D$ system to iso-

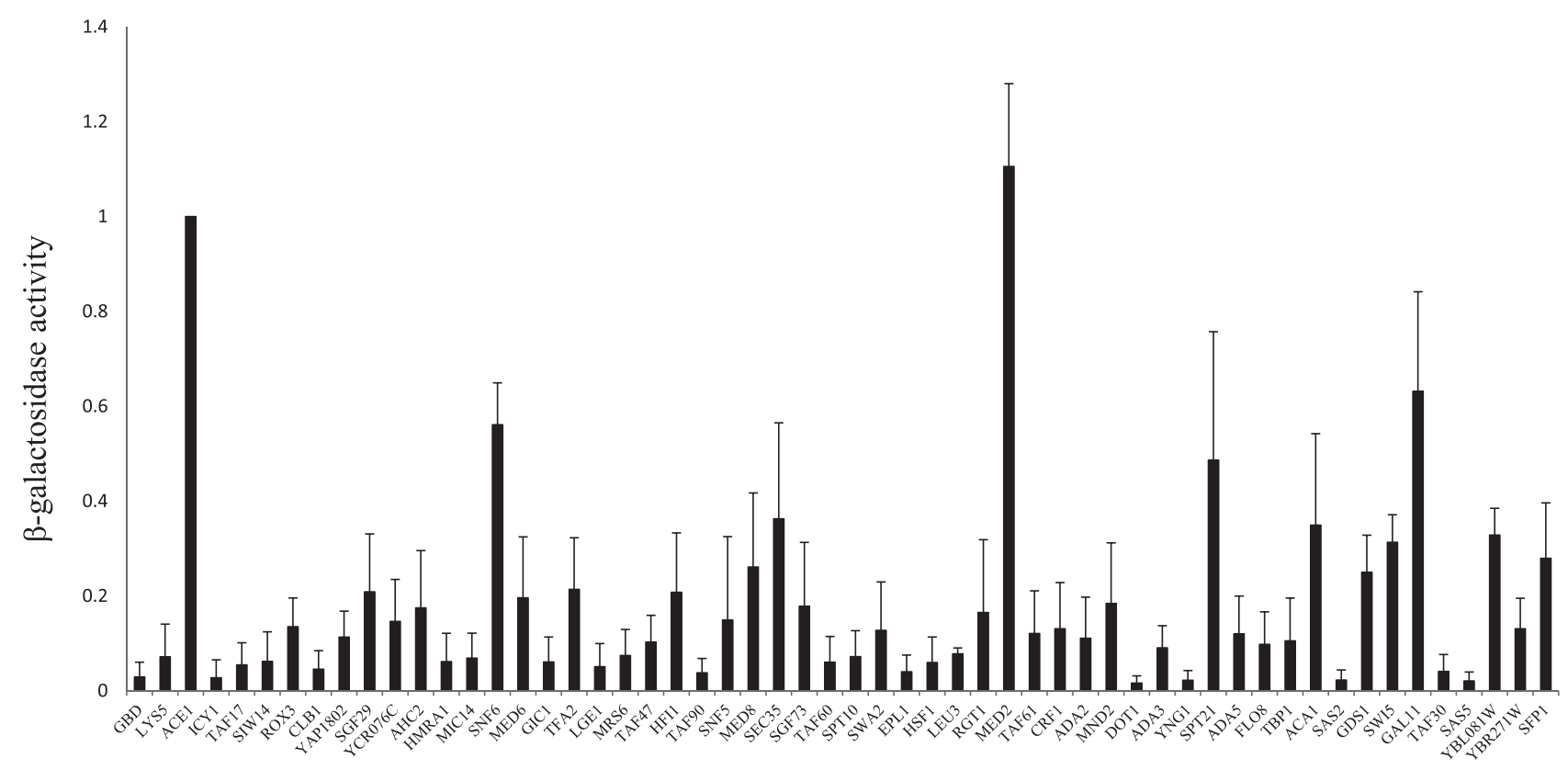

Gene name

Fig. 1. Measurement of the transcriptional activity of boundary elements. The transcriptional activity of 55 boundary elements was assessed by a one-hybrid assay. FUY49 was transformed with plasmids harboring each boundary element fused to GBD (see Supplementary Table S2). The x-axis shows the 55 boundary genes, and the y-axis shows $\beta$-galactosidase activity. Transcriptional activity was normalized against that of ACE1. Error bars show standard deviation of three independent experiments. 
late boundary proteins among all $S$. cerevisiae genes, we next investigated where Gic1 has boundary function in vivo by performing ChIP analysis with an anti-Sir3 antibody. If Gic1 functions at boundaries in some regions, occupancy of Sir3 around these regions should be increased in the gic1 1 strain. In $S$. cerevisiae, $H M R$, $H M L$, telomeric regions of chromosome ends, and rDNA are well-known heterochromatic regions, and boundary regions may be located close to them (Fourel et al., 1999; Donze and Kamakaka, 2001; Bi, 2002; Oki and Kamakaka, 2005). We analyzed $H M R$, telomeric and rDNA regions (Fig. 2). First, we focused on the $H M R$ region and compared Sir3 occupancy between wild type (WT) and gic1 $\Delta$ strains. Sir3 occupancy was increased at boundary regions ( $\mathrm{R} 7$ and $\mathrm{R} 10$ ) located on either side of the $H M R$, in gic1 $\Delta$ (Fig. 2, a and b). These data suggest that Gic1

(a)

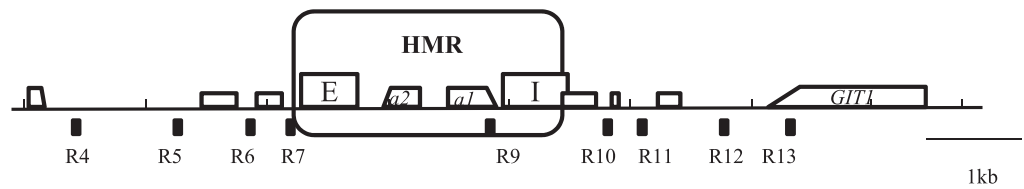

(b)

(c)

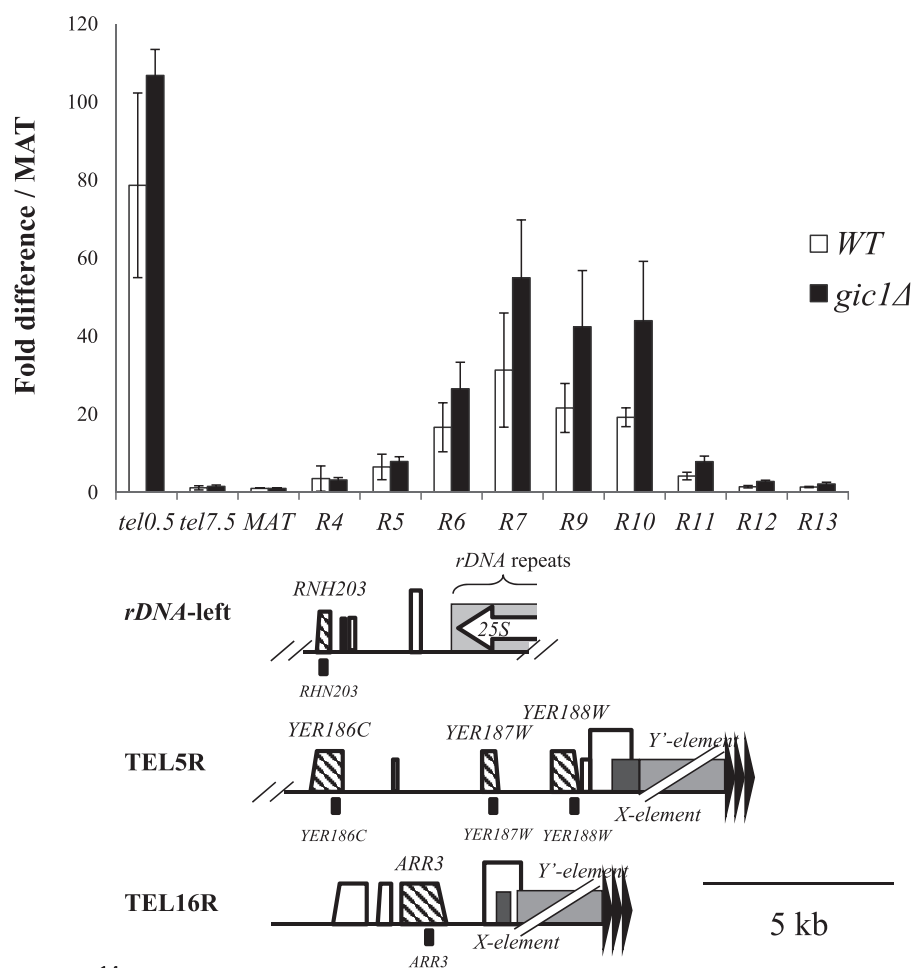

(d)

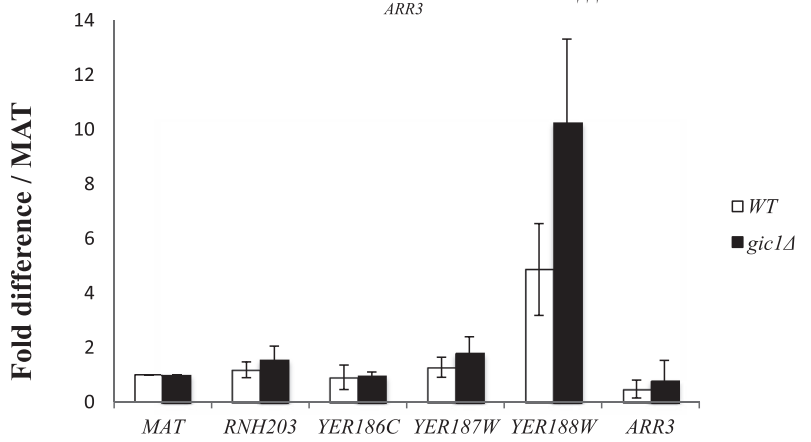

Fig. 2. Gic1 affects heterochromatin boundaries at $H M R$ and telomeric regions. We compared the distribution of Sir3 between WT (FUY31) and gic1 (FUY53) strains at HMR and telomeric regions by performing a ChIP assay. (a) Schematic diagram of the HMR region. R4-R13 indicate the primer sets used for the ChIP assay. (b) Results of the ChIP assay for the HMR region. The positive control is tel0.5 and the negative control is tel7.5. (c) Schematic diagram of telomeres. Black points denote the primer sets used for the ChIP assay. (d) Results of the ChIP assay with WT (white bars) and gic1s (black bars) strains at telomeres. The x-axis shows the primer set used. The y-axis shows Sir3 distribution. Data are normalized against that of $M A T$. The error bars show standard deviation of three independent experiments. 
has a boundary function in the $H M R$ region. In addition, its level at the $H M R$ core region (R9) was also increased. The reason for this effect is unclear. Gic1 may have a function in stabilizing the chromatin association of Sir3, in addition to its role in boundary formation.

Next, we assessed telomeric and rDNA regions (Fig. 2c). YER187W, YER188W and YER186C are located close to the right telomere of chromosome $\mathrm{V}$, and $A R R 3$ is located close to the right telomere of chromosome XVI. Sir3 occupancy at YER188W was higher in the gic1 $\triangle$ strain, but not appreciably different at the other regions (Fig. 2d). These data suggest that Gic1 also has a boundary function close to $Y E R 188 \mathrm{~W}$ in the right arm of telomere $\mathrm{V}$, similar to its boundary function in the $H M R$ region.

Gic1 binds to histones and functions at boundaries independent of other boundary proteins Having detected boundary activity of Gic1 at HMR and telomeric regions using the ChIP assay, we next investigated whether Gic1 can bind to nucleosomes. Gic1 reportedly localizes to both the nucleus and the cytoplasm (Chen et al., 1997; Hofken and Schiebel, 2004; Takahashi and Pryciak, 2007). However, it has not been reported whether Gic1 can bind to nucleosomes. To investigate this, we performed two-hybrid analysis with histone $\mathrm{H} 2 \mathrm{~A}$, histone H2B, histone H3, histone $\mathrm{H} 4$ and Sir3. In this assay, FUY49 (for one- or two-hybrid assay strains, see Supplementary Table S1) was transformed with GADand GBD-fusion plasmids (Supplementary Table S2).
Gic1 could bind to all histones, but not to Sir3 (Fig. 3). These data suggest that Gic1 binds to histones directly and has boundary function.

Next, we investigated whether Gic1 works together with other boundary proteins to form a boundary. We selected 11 boundary proteins (Ada1, Ada2, Sas2, Sas5, Dot1, Snf6, Taf47, Med2, Clb1, Ycr076c and Gds1), which were in each of the categories in our screening. Each of these proteins retained boundary activity in the gic1 $\Delta$ strain, meaning that the boundary activity of Gic1 is independent of other boundary proteins (Supplementary Fig. S1).

Gic1 has two small boundary domains We performed deletion analysis of Gic1 to identify the minimum region(s) necessary for its boundary function, using the patch mating system used for boundary screening (Oki et al., 2004) (Fig. 4a). A low level or absence of mating indicated boundary activity (Fig. 4b). First, we divided Gic1 into two regions, its N-terminal domain (1-151 amino acids (aa)), which includes the CRIB domain, and its Cterminal domain (167-315 aa). The CRIB domain in the $\mathrm{N}$-terminal domain did not appear to have any role in Gic1 boundary function. A series of Gic1 deletion mutants of the C-terminal domain were constructed and tested using patch mating. These data indicated that 228-241 aa and 283-302 aa of Gic1 had boundary activity. To confirm this, we generated strains in which these regions were deleted $(\Delta 228-241$ aa and $\Delta 283-302$

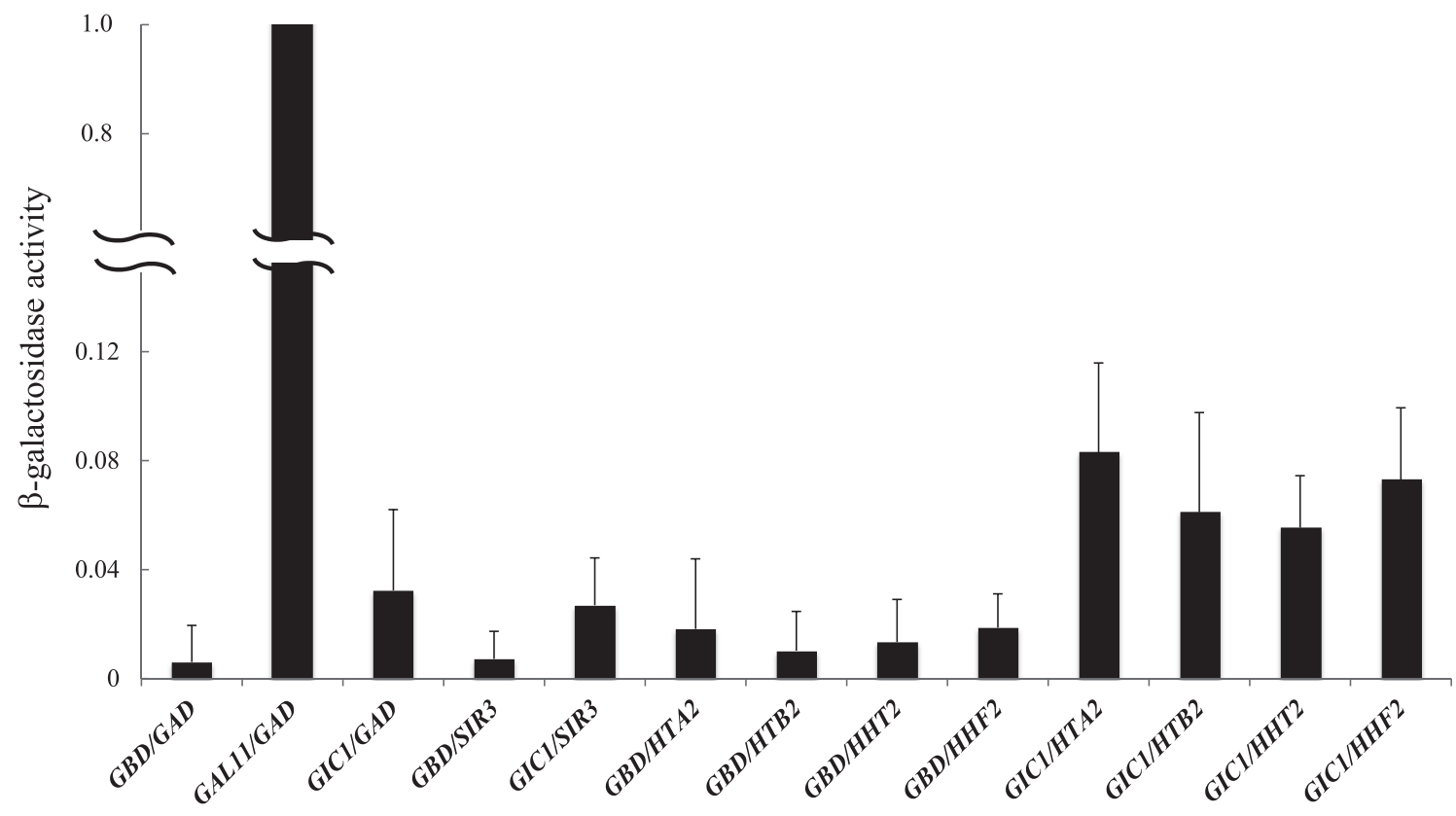

Gene name

Fig. 3. Gic1 associates with histones. GIC1 and GAL11 were fused to GBD. Histones and SIR3 were fused to GAD (see Supplementary Table S2). FUY49 was transformed with these plasmids. The $\mathrm{x}$-axis shows each combination. The $\mathrm{y}$-axis shows $\beta$-galactosidase activity. Data were normalized against those obtained using a combination of GBD-GAL11 and GAD. 


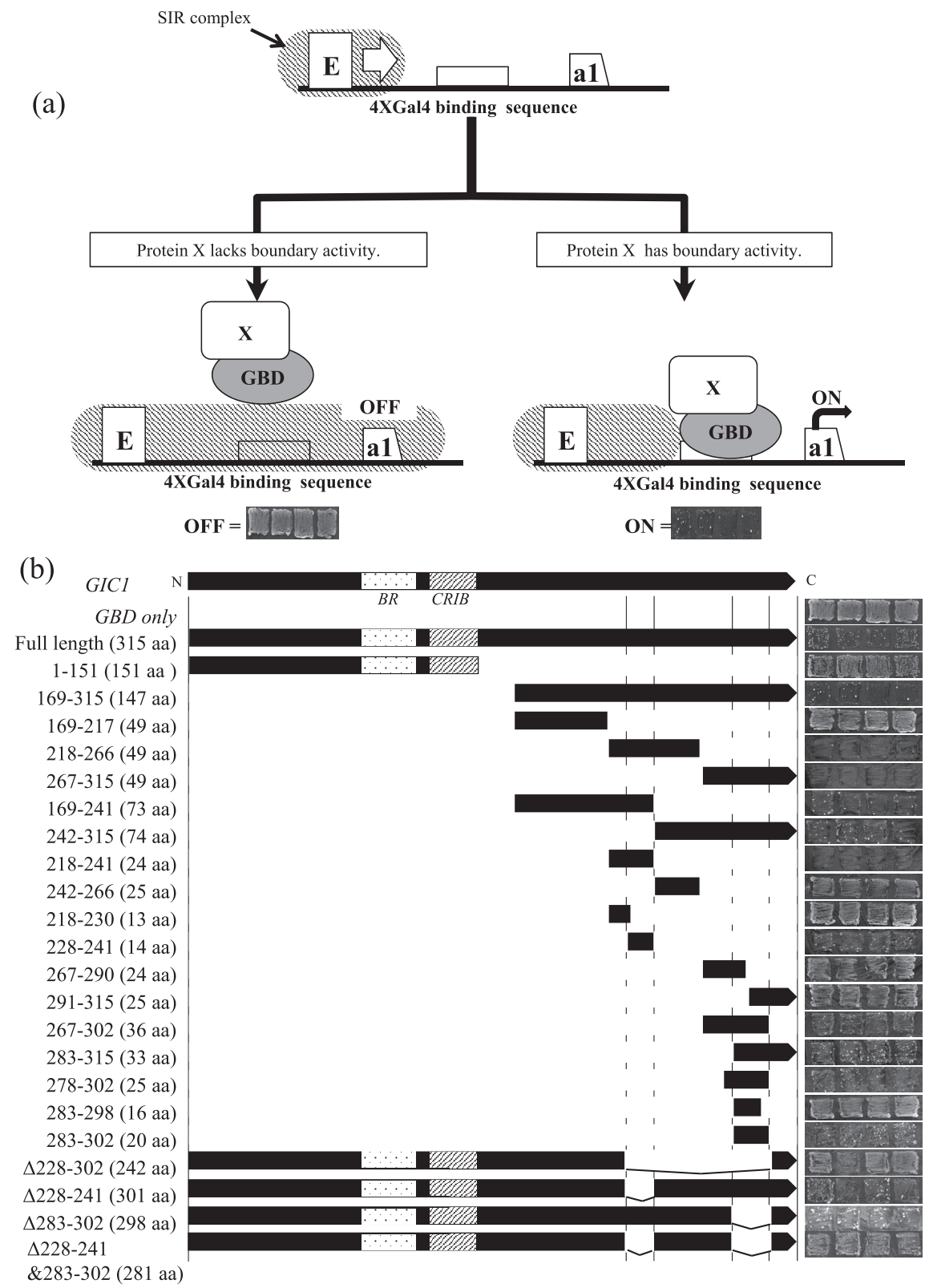

Fig. 4. Identification of the minimum boundary domains of Gic1. (a) Schematic view of our boundary screening system. If protein $\mathrm{X}$ does not have boundary activity, the yeast can grow on the selection plate (OFF). If protein $\mathrm{X}$ has boundary activity, the yeast cannot grow on the selection plate (ON). For details, see our previous paper (Oki et al., 2004). (b) Plasmids were constructed harboring various domains of Gic1 and a mating assay was performed. The plasmids are listed in Supplementary Table S2. The numbers on the left denote the aa of Gic1. Black boxes denote domains, boxes containing slanted lines denote the CRIB domain, and boxes containing dots denote the BR domain. The right panel shows the mating results with four independent colonies.

aa) and performed patch mating. These strains did not have boundary activity. If endogenous Gic1 forms a homodimer with these two minimum regions, this would explain these data and mean that their function is not related to boundaries. When we performed the same experiment with the gic1 $\Delta$ strain, both domains retained boundary function (Fig. 5). Next, we investigated why Gic1 has two domains that are important for its boundary function and whether the same proteins can bind to both domains. We performed mass spectrometry to identify binding proteins of the boundary domains (228-241 aa and 283-302 aa) by constructing strains expressing the minimum domains tagged with G196 (a gift from Dr. T. Urano) (Kamata et al., 2013). However, we could not identify a protein that specifically binds to these domains (data not shown). The level of Gic1 was low in cells; 


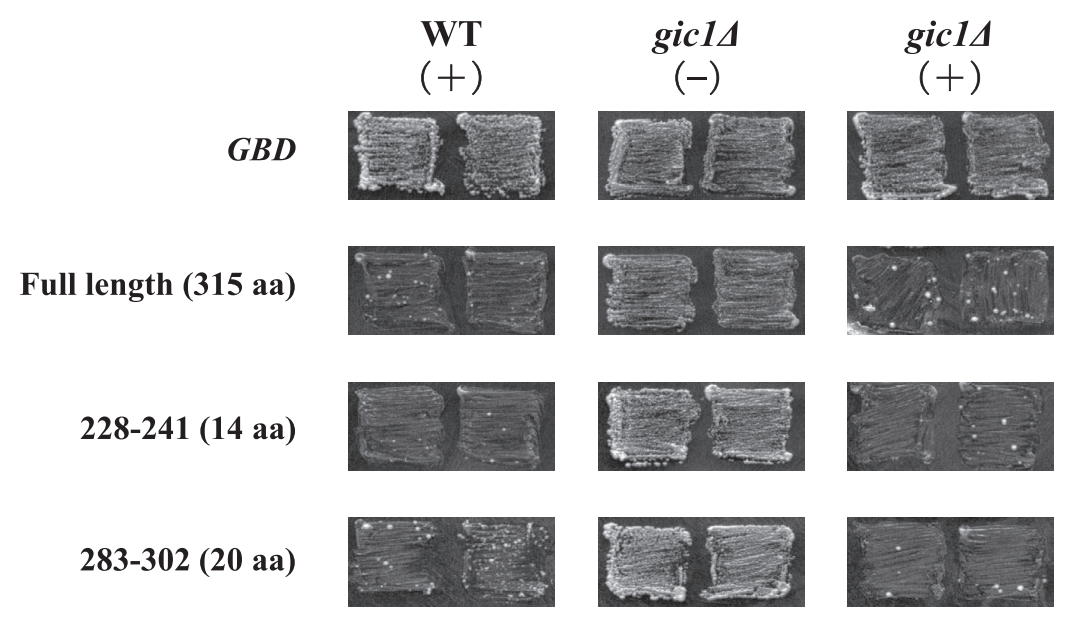

Fig. 5. The two minimum boundary domains of Gic1 form a boundary by themselves. ROY2042 (Gal4bd+), FUY163 (gic14, Gal4bd-) and FUY164 (gic14, Gal4bd+) were transformed with a plasmid harboring one of the minimum boundary domains, and a mating assay was performed.

therefore, Gic1 may not have been detectable under the conditions used for affinity purification.

\section{DISCUSSION}

Gic1 has a novel boundary function in vivo In this report, we investigated the novel boundary protein Gic1. Gic1 has boundary activity according to a mating assay; however, its boundary function in vivo is unknown (Oki et al., 2004). First, we attempted to identify transcription-independent boundary genes among 55 genes by performing a one-hybrid assay (Reynolds et al., 2001). This showed that 11 genes had a transcription-associated boundary function and 44 genes had a transcriptionindependent boundary function. Some of these 44 genes were previously reported to function at boundaries. For example, Sas2, the best-known boundary protein, prevents the deacetylase activity of Sir2 (Kimura et al., 2002; Suka et al., 2002). Sgf29 and Sgf73 are components of the SAGA, SAGA-like, ADA and HAT-2 complexes (Kamata et al., 2013, 2014). GIC1 does not belong to the Histone modifications, Transcription factors or Cell cycle groups. Our data suggest that Gic1 has novel boundary function by working together with another protein. Therefore, we performed liquid chromatography tandem mass spectrometry (LC-MS/MS) analysis to identify a novel binding protein; however, we could not detect a novel binding partner of Gic1 (data not shown). We speculate that the interactions between Gic1 and such proteins are very weak or very transient, or that the concentration of such complexes is low.

Gic1 has boundary function in vivo Next, we showed that Gic1 has native boundary activity at $H M R$ and telomere sites. Gic1 has many basic residues, notably the BR domain (29-33 aa). The BR domain is located close to the CRIB domain and is involved in proteinprotein and protein-membrane interactions and localization of nuclear-targeting activity (Takahashi and Pryciak, 2007). Therefore, we predicted that the nuclear localization of Gic1 prevents heterochromatin formation in vivo. ChIP analysis using an anti-Sir3 antibody revealed the boundary function of Gic1 at $H M R$ and telomeric regions. Elongation of heterochromatin from $H M R$-left to R7-R6, from $H M R$-right to R10-R11 and from the telomere to YER188W was not prevented in the gic1A strain (Fig. 2). However, the function of Gic1 in histone modifications or transcription regulation is unknown. We confirmed the novel heterochromatin boundary function of Gic1. We next investigated whether Gic1 can bind directly to these boundary regions. We performed ChIP with a strain expressing Flag-Gic1; however, we could not detect Gic1 at these boundary regions (data not shown), although in our previous boundary screening study Gic1 had boundary activity. Given that Gic1 has many functions in living cells, the level of Gic1 at boundary regions may be low.

Gic1 interacts with histones Gic1 exhibits a genetic interaction with Cdc42, Rsr1, Bem1, Cdc24 and Bem2 (Chen et al., 1997; Johnson, 1999; Kawasaki et al., 2003; Gandhi et al., 2006). These proteins are associated with bud emergence, actin and septin localization, and mitosis. However, no reports have shown an interaction between Gic1 and histones. Such interactions are very important to form a heterochromatin boundary; therefore, we performed two-hybrid analysis (Fig. 3). Our results suggest that Gic1 weakly interacts with histones and that this is related to heterochromatin boundaries.

Gic1 has two minimum boundary domains Some boundary proteins have several functional boundary 
domains and interact with other proteins (Hatanaka et al., 2011; Kamata et al., 2014). We hypothesized that Gic1 also has boundary domains. We performed domain analysis using a mating assay to identify the boundary domain(s) of Gic1 and found two minimum boundary domains (Fig. 4b). These two domains do not contain the CRIB or BR domains, which assist budding at the neck and nuclear localization of Gic1. It is suggested that Gic1 has a novel function to prevent elongation of heterochromatin in relation to histone modifications. Moreover, we checked the ability of the two minimum boundary domains to protect a1 gene transcription from heterochromatin elongation using the gic1s strain. If Gic1 forms a homodimer, these two minimum domains might bind to and recruit intact Gic1. Both domains were independently capable of preventing heterochromatin elongation in the gic1s strain (Fig. 5). Next, we investigated how the two minimum domains function in the boundary activity of full-length Gic1 by generating gic1 mutants in which each minimum boundary domain was disrupted. Interestingly, the mutant in which both domains were disrupted did not have boundary activity, whereas those in which only one domain was disrupted had weak boundary activity (Fig. 4b). These data suggest that these two minimum boundary domains work together to assemble a protein complex required for boundary formation. Therefore, we performed LC-MS/ MS analysis with strains expressing FLAG-tag fusion proteins to identify proteins that bind to these minimum boundary domains. We identified some weak binding proteins, one of which was Spa2, which is a component of the polarisome and can bind to Sir2 (Liu et al., 2010). The Gic1-Spa2-Sir2 complex may be important for boundary activity; however, further studies are required to prove this.

Gic1 associates with Zds1, which affects cell polarity and transcriptional silencing (Bi and Pringle, 1996; Zanelli and Valentini, 2005). In the $z d s 1$ mutant, HM region and rDNA silencing is up-regulated, whereas telomere silencing is down-regulated (Roy and Runge, 2000). Moreover, rDNA recombination and life span are affected in the $z d s 1$ mutant. We hypothesized that Gic1 might work together with Zds1 for boundary activity. Therefore, we performed the boundary assay with the minimum Gic1 boundary domain in the $z d s 1 \Delta$ strain. However, boundary activity was retained, indicating that Gic1 does not work together with Zds1 to form a boundary (Supplementary Fig. S2).

Gic1 interacts with many proteins and has many functions in vivo. More experiments need to be performed in order to elucidate the native boundary mechanism in which Gic1 functions.

We thank Dr. J. Rine, Dr. R. T. Kamakaka, Dr. T. Tsukiyama, Dr. N. Hayashi, Dr. H. Kato and Dr. T. Iida for providing the yeast strains and plasmids. We also thank Dr. T. Urano for providing the anti-G196 antibody.

\section{REFERENCES}

Bi, E., and Pringle, J. R. (1996) ZDS1 and ZDS2, genes whose products may regulate Cdc42p in Saccharomyces cerevisiae. Mol. Cell. Biol. 16, 5264-5275.

$\mathrm{Bi}, \mathrm{X}$. (2002) Domains of gene silencing near the left end of chromosome III in Saccharomyces cerevisiae. Genetics 160, 1401-1407.

Brown, J. L., Jaquenoud, M., Gulli, M. P., Chant, J., and Peter, M. (1997) Novel Cdc42-binding proteins Gic1 and Gic2 control cell polarity in yeast. Genes Dev. 11, 2972-2982.

Burbelo, P. D., Drechsel, D., and Hall, A. (1995) A conserved binding motif defines numerous candidate target proteins for both Cdc42 and Rac GTPases. J. Biol. Chem. 270, 29071-29074.

Chen, G. C., Kim, Y. J., and Chan, C. S. (1997) The Cdc42 GTPase-associated proteins Gic1 and Gic2 are required for polarized cell growth in Saccharomyces cerevisiae. Genes Dev. 11, 2958-2971.

Donze, D., and Kamakaka, R. T. (2001) RNA polymerase III and RNA polymerase II promoter complexes are heterochromatin barriers in Saccharomyces cerevisiae. EMBO J. 20 , $520-531$.

Donze, D., Adams, C. R., Rine, J., and Kamakaka, R. T. (1999) The boundaries of the silenced HMR domain in Saccharomyces cerevisiae. Genes Dev. 13, 698-708.

Fourel, G., Revardel, E., Koering, C. E., and Gilson, E. (1999) Cohabitation of insulators and silencing elements in yeast subtelomeric regions. EMBO J. 18, 2522-2537.

Gandhi, M., Goode, B. L., and Chan, C. S. (2006) Four novel suppressors of gic1 gic2 and their roles in cytokinesis and polarized cell growth in Saccharomyces cerevisiae. Genetics 174, $665-678$.

Hatanaka, A., Chen, B., Sun, J. Q., Mano, Y., Funakoshi, M., Kobayashi, H., Ju, Y., Mizutani, T., Shinmyozu, K., Nakayama, J., et al. (2011) Fub1p, a novel protein isolated by boundary screening, binds the proteasome complex. Genes Genet. Syst. 86, 305-314.

Hofken, T., and Schiebel, E. (2004) Novel regulation of mitotic exit by the Cdc42 effectors Gic1 and Gic2. J. Cell Biol. 164, 219-231.

Imai, S., Armstrong, C. M., Kaeberlein, M., and Guarente, L. (2000) Transcriptional silencing and longevity protein Sir2 is an NAD-dependent histone deacetylase. Nature $\mathbf{4 0 3}$, 795-800.

Ito, T., Tashiro, K., Muta, S., Ozawa, R., Chiba, T., Nishizawa, M., Yamamoto, K., Kuhara, S., and Sakaki, Y. (2000) Toward a protein-protein interaction map of the budding yeast: A comprehensive system to examine two-hybrid interactions in all possible combinations between the yeast proteins. Proc. Natl. Acad. Sci. USA 97, 1143-1147.

Iwase, M., Luo, J., Nagaraj, S., Longtine, M., Kim, H. B., Haarer, B. K., Caruso, C., Tong, Z., Pringle, J. R., and Bi, E. (2006) Role of a Cdc42p effector pathway in recruitment of the yeast septins to the presumptive bud site. Mol. Biol. Cell 17, 1110-1125.

Jaquenoud, M., and Peter, M. (2000) Gic2p may link activated Cdc42p to components involved in actin polarization, including Bni1p and Bud6p (Aip3p). Mol. Cell. Biol. 20, 62446258.

Johnson, D. I. (1999) Cdc42: An essential Rho-type GTPase controlling eukaryotic cell polarity. Microbiol. Mol. Biol. Rev. 
63, 54-105.

Kamata, K., Hatanaka, A., Goswami, G., Shinmyozu, K., Nakayama, J., Urano, T., Hatashita, M., Uchida, H., and Oki, M. (2013) C-terminus of the Sgf73 subunit of SAGA and SLIK is important for retention in the larger complex and for heterochromatin boundary function. Genes Cells 18, 823-837.

Kamata, K., Goswami, G., Kashio, S., Urano, T., Nakagawa, R., Uchida, H., and Oki, M. (2014) The N-terminus and Tudor domains of Sgf29 are important for its heterochromatin boundary formation function. J. Biochem. 155, 159-171.

Kawasaki, R., Fujimura-Kamada, K., Toi, H., Kato, H., and Tanaka, K. (2003) The upstream regulator, Rsr1p, and downstream effectors, Gic1p and Gic2p, of the Cdc42p small GTPase coordinately regulate initiation of budding in Saccharomyces cerevisiae. Genes Cells 8, 235-250.

Kimura, A., Umehara, T., and Horikoshi, M. (2002) Chromosomal gradient of histone acetylation established by Sas2p and Sir2 $p$ functions as a shield against gene silencing. Nat. Genet. 32, 370-377.

Landry, J., Sutton, A., Tafrov, S. T., Heller, R. C., Stebbins, J., Pillus, L., and Sternglanz, R. (2000) The silencing protein SIR2 and its homologs are NAD-dependent protein deacetylases. Proc. Natl. Acad. Sci. USA 97, 5807-5811.

Lindsey, R., Ha, Y., and Momany, M. (2010) A septin from the filamentous fungus $A$. nidulans induces atypical pseudohyphae in the budding yeast $S$. cerevisiae. PLoS One 5, e9858.

Liu, B., Larsson, L., Caballero, A., Hao, X., Oling, D., Grantham, J., and Nystrom, T. (2010) The polarisome is required for segregation and retrograde transport of protein aggregates. Cell 140, 257-267.

Loo, S., and Rine, J. (1995) Silencing and heritable domains of gene expression. Annu. Rev. Cell Dev. Biol. 11, 519-548.

Moazed, D. (2001) Common themes in mechanisms of gene silencing. Mol. Cell 8, 489-498.

Moazed, D., Rudner, A. D., Huang, J., Hoppe, G. J., and Tanny, J. C. (2004) A model for step-wise assembly of heterochromatin in yeast. Novartis Found. Symp. 259, 48-56; discussion 56-62, 163-9.

Oki, M., and Kamakaka, R. T. (2002) Blockers and barriers to transcription: competing activities? Curr. Opin. Cell Biol. 14, 299-304.

Oki, M., and Kamakaka, R. T. (2005) Barrier function at $H M R$. Mol. Cell 19, 707-716.
Oki, M., Valenzuela, L., Chiba, T., Ito, T., and Kamakaka, R. T. (2004) Barrier proteins remodel and modify chromatin to restrict silenced domains. Mol. Cell. Biol. 24, 1956-1967.

Reynolds, A., Lundblad, V., Dorris, D., and Keaveney, M. (2001) Yeast vectors and assays for expression of cloned genes. Curr. Protoc. Mol. Biol. Chapter 13, Unit13.6.

Roy, N., and Runge, K. W. (2000) Two paralogs involved in transcriptional silencing that antagonistically control yeast life span. Curr. Biol. 10, 111-114.

Rusche, L. N., Kirchmaier, A. L., and Rine, J. (2003) The establishment, inheritance, and function of silenced chromatin in Saccharomyces cerevisiae. Annu. Rev. Biochem. 72, 481516.

Sadian, Y., Gatsogiannis, C., Patasi, C., Hofnagel, O., Goody, R. S., Farkasovsky, M., and Raunser, S. (2013) The role of Cdc42 and Gic1 in the regulation of septin filament formation and dissociation. Elife 2, e01085.

Simms, T. A., Dugas, S. L., Gremillion, J. C., Ibos, M. E. Dandurand, M. N., Toliver, T. T., Edwards, D. J., and Donze, D. (2008) TFIIIC binding sites function as both heterochromatin barriers and chromatin insulators in Saccharomyces cerevisiae. Eukaryot. Cell 7, 2078-2086.

Suka, N., Luo, K., and Grunstein, M. (2002) Sir2p and Sas2p opposingly regulate acetylation of yeast histone $\mathrm{H} 4$ lysine 16 and spreading of heterochromatin. Nat. Genet. 32, 378383.

Sun, J. Q., Hatanaka, A., and Oki, M. (2011) Boundaries of transcriptionally silent chromatin in Saccharomyces cerevisiae. Genes Genet. Syst. 86, 73-81.

Takahashi, S., and Pryciak, P. M. (2007) Identification of novel membrane-binding domains in multiple yeast Cdc42 effectors. Mol. Biol. Cell 18, 4945-4956.

Tanny, J. C., Dowd, G. J., Huang, J., Hilz, H., and Moazed, D. (1999) An enzymatic activity in the yeast Sir2 protein that is essential for gene silencing. Cell 99, 735-745.

Triolo, T., and Sternglanz, R. (1996) Role of interactions between the origin recognition complex and SIR1 in transcriptional silencing. Nature 381, 251-253.

Valenzuela, L., Dhillon, N., and Kamakaka, R. T. (2009) Transcription independent insulation at TFIIIC-dependent insulators. Genetics 183, 131-148.

Zanelli, C. F., and Valentini, S. R. (2005) Pkc1 acts through Zds1 and Gic1 to suppress growth and cell polarity defects of a yeast eIF5A mutant. Genetics 171, 1571-1581. 